\title{
OFERTA CIENTÍFICO-TECNOLÓGICA DE LA MACRO REGIÓN CENTRO SUR DE CHILE Y SU RELACIÓN CON LOS SISTEMAS PRODUCTIVOS REGIONALES
}

\section{Ciencia, tecnología e innovación en el escenario competitivo contemporáneo}

Tres ejes teóricos permiten reseñar el foco analítico. En primer lugar, la comprensión del desarrollo de la ciencia, tecnología e innovación para la competitividad. En segundo, la construcción de la capacidad científica, tecnológica y de innovación. Y en tercer lugar, interesa abordar la comprensión y gestión del desarrollo de las redes socioproductivas del territorio, funcionales al desarrollo de la innovación.

Conocimiento, ciencia, tecnología e innovación en la construcción de ventajas competitivas

Tradicionalmente, el desarrollo del comercio internacional y su relación con el crecimiento y progreso económico se ha fundamentado, en gran parte, en la teoría de la ventaja comparativa, en la cual la estructura económico-productiva especializada en bienes y servicios que se pueden efectuar se expresa en costos relativamente más bajos. En esta perspectiva, la lógica de la ventaja comparativa en un patrón de eficiencia que implica especializar la producción en sectores que cuentan con una adecuada dotación de inicio de factores productivos, generalmente de orden geográfico-espacial, ajenos a una construcción social y económica de factores (Gatica, 2002). Este modelo explica el patrón de especialización internacional de cada país en función de la disponibilidad y costo relativo de recursos productivos.

No obstante su validez empírica, dicho planteamiento ha encontrado su contraste en la ventaja competitiva, planteando que 
el costo relativo de factores constituye una prerrogativa de orden inferior, susceptible de ser reproducida o superada por la innovación. En tanto, la ventaja competitiva sostenible, de orden superior, estaría ligada a la capacidad de ofrecer un valor superior en la producción, a la vez que singular en términos de calidad, características especiales y servicios asociados a ésta (Porter, 1987). Como tal, no obstante, demanda capacidades avanzadas, que se construyen a partir de un flujo de inversiones generadoras de procesos de aprendizaje y que permiten incrementos de productividad, fundamento último de la competitividad (Millán, 1994).

Surgen así mercados que obedecen a dos posibles caracterizaciones, en las cuales la competitividad está determinada por el costo de factores o bien por la capacidad de diferenciación e innovación, explicada por el flujo de inversiones y sus procesos de aprendizaje vinculables. Al respecto, las ventajas comparativas se visibilizan cada vez más como factores de orden inferior, de imitación o superación relativamente fácil, frente a avances técnicos, acrecencia del conocimiento y extensión de los flujos de información, aspectos que impulsan la construcción de nuevos factores y cimientan una posición superior de los productores (Porter, 1996). Situación que se explica porque aquella hace abstracción del avance tecnológico, factor cardinal en los incrementos de productividad y que, lejos de hacer ineficiente la producción de nuevos bienes, ha permitido una reducción notable en costos de producción (Muñoz, 2001).

Por lo tanto, desde esta nueva perspectiva, la competitividad de las empresas está determinada centralmente por su capacidad de innovación y, por lo tanto, la clave estratégica reside en promover factores que la estimulen. Al respecto, diversos estudios han demostrado que la innovación, especialmente tecnológica (Pérez, 2001), explica patrones de comercio y comportamientos competitivos; así, la construcción de ventajas competitivas es finalmente un acto de innovación, incluyendo avances tecnológicos, surgimiento de nuevos insumos, formas de organizar la producción, descubrimiento de demandas, aparición de potenciales de mercado y estilos de comercialización. 
De este modo, los avances tecnológicos e innovaciones se producen por la acción de actores altamente capacitados, estratégicamente activos en procesos de investigación, desarrollo e invención y el punto central reside entonces en impulsar factores que incentiven el esfuerzo creador. Al respecto, se observa que el proceso de construcción de ventajas competitivas e innovación se encuentra fuertemente mediatizado por el mercado, en el que actúan cinco fuerzas competitivas que impulsan el proceso estratégico innovador de las empresas: rivalidad de la industria, amenaza de potenciales entrantes, de productos sustitutivos, poder de negociación con consumidores y proveedores. Según sea la intensidad con que operan estas fuerzas, los actores se verán impulsados a modernizar. En el fondo, el grado de exigencia del entorno y las amenazas del mercado actúan como un aliciente poderoso para el impulso innovador (Porter, 1979), es decir, la presión del mercado y las exigencias de los consumidores son fuentes generadoras de aprendizaje y creación.

Los factores que incentivan el proceso innovador e inciden en la capacidad de aprendizaje son, por una parte, la elevada exposición al cambio y amplitud de agentes y actividades generadoras de complejidad productiva; por otra, la capacidad para absorber el cambio y traducirlo en bienes, servicios y estrategias concretas. En este contexto, el mercado y sus presiones competitivas surgen como aspectos especialmente incentivadores del proceso innovativo para las empresas.

\section{Construcción de capacidades competitivas sobre la base de ciencia, tecnología e innovación}

La comprensión del proceso de construcción de ventajas competitivas sobre la base de la ciencia, tecnología e innovación pone en primer lugar el hecho de que, si bien la competencia y la producción son cada vez más globales, es imperativo que las capacidades que permiten insertarse en la competencia general se construyan localmente (Rojas, 2001). Esta idea sitúa al territorio como plataforma para la construcción de capacidades competitivas, puesto que cuenta con ventajas de un entorno de mayor complejidad y singularidad, que incentiva al cambio y la mejora continua, en tanto que difunde el 
conocimiento y sus resultados. Todo lo cual fortalece el territorio en un sentido articulado y complejo (Galdames et al., 2002).

Al respecto, la evidencia indica que el éxito competitivo se encuentra circunscrito a localidades específicas, generalmente concentrado en áreas geográficas identificables y muchas veces de reducida extensión espacial. Esto, dado que la existencia concentrada de competidores, clientes, proveedores y servicios especializados en un área geográfica reducida genera un clima propicio para la mejora continua e innovación, con líneas de comunicación cortas y presiones competitivas sentidas agudamente. En el fondo, la habilidad de crear una concentración única de productores, proveedores, clientes y servicios conexos de alta calidad y productividad genera condiciones especialmente impulsoras para el incremento de la competitividad.

Ahora bien, de la gama de posibles explicaciones de una alta concentración productiva en un sector geográfico determinado se han distinguido elementos especialmente inductores del establecimiento de empresas en un territorio determinado (Millán, 1994):

- Existencia de factores atractivos básicos en cuanto a su relación calidad-costo.

- Dotación de una infraestructura adecuada y una ubicación óptima.

- Dotación de factores especializados.

Si bien son los productores quienes compiten directamente en los mercados, los territorios desempeñan el papel de plataforma que aporta las condiciones de entorno para el incremento de la competitividad, mediante un sistema en permanente actividad que obra de manera simultánea, acumulativa, interdependiente y autorreforzante por la concurrencia sinérgica de distintos actores.

Sobre el carácter sistémico y complejo de la construcción competitiva a partir de la innovación, una visión conceptual señala que el desarrollo de ésta depende del fortalecimiento de los diamantes competitivos territoriales. Esto significa que el éxito económico y productivo se relaciona con la calidad y fortaleza de cuatro atributos genéricos que conforman territorio (Porter, 1991): 
- Condiciones de los factores productivos.

- Condiciones de la demanda.

- Sectores afines y de apoyo.

- Estrategia, estructura y rivalidad de los sectores.

Conformándose un cuadro en el cual la competitividad es el resultado de un sistema interactivo y complejo, en el que las partes del diamante competitivo territorial se refuerzan mutuamente y, por lo tanto, conforman una primera aproximación a la visión sistémica de la competitividad, y en el que, por lo demás, la ciencia y tecnología influyen poderosa y transversalmente con especial intensidad en las condiciones de los factores productivos y el desarrollo de sectores de apoyo.

Otra línea argumental ubica a la construcción competitiva como el resultado de la organización social, en cuyo seno se generan ventajas competitivas en función de la interacción de múltiples parámetros de relevancia. De esta forma, la competitividad resulta de la relación en cuatro niveles sistémicos: meta, macro, meso y micro (Esser et al., 1994) que de manera más específica indican:

- Nivel Meta: en el cual el consenso social respecto de una política económica dirigida al mercado, un patrón básico de organización social que aglutine las fuerzas de los agentes y la disposición y capacidad para implementar estrategias con miras al desarrollo competitivo son fundamentales y, por lo tanto, se apunta a una elevada capacidad de organización, interacción y estrategia, por parte de los agentes.

- Nivel Macro: en el que un marco macroeconómico estable, una política de competencia y una política comercial orientada a la exportación son fundamentales y, por lo tanto, se apunta a crear condiciones generales para una competencia eficaz que presione a las empresas para el incremento de la productividad, la innovación y la competitividad.

- Nivel Meso: en el que es preciso reformar la infraestructura con miras a la competitividad, así como también las políticas específicas dirigidas a la educación, ciencia y tecnología son fundamentales y, por lo tanto, se orienta a configurar el entorno específico de las empresas con miras al incremento del potencial competitivo. 
- Nivel Micro: en el cual lo determinante es una gestión efectiva de las innovaciones técnicas y organizacionales por parte de las empresas, siendo la gestión tecnológica eficaz un requisito para obtener innovaciones continuas en productos y procesos.

Los niveles descritos, si bien se presentan bajo una óptica complementaria e insustituible, en la perspectiva del desarrollo de la competitividad se sugiere al nivel meso en un primer orden de importancia, puesto que en éste se van plasmando los procesos de aprendizaje colectivo que le entregan al sistema un valor difícilmente imitable por los competidores y es donde radica la formación de un entorno capaz de fomentar, completar y multiplicar los esfuerzos de los agentes económico productivos (Menéndez, 2002).

De acuerdo con ello, la dinámica del desarrollo competitivo dependerá centralmente del desarrollo científico y tecnológico $y$, fundamentalmente, del contacto estrecho entre universidades, institutos científico-tecnológicos, agencias de información para la exportación, empresas y otros agentes quienes, en su interacción, generan procesos acumulativos de aprendizaje, funcionales a la formación de redes de colaboración interagentes y circunscritos a un territorio determinado.

En este escenario, el papel de las universidades e instituciones de educación superior reside en que la innovación, que influye directamente sobre el ritmo y naturaleza del desarrollo, impone nuevos requisitos como formación adecuada de recursos humanos. A su vez, la universidad se constituye en la principal institución para introducir y difundir los lineamientos doctrinarios y teóricos que se complementan con el paradigma tecnológico, suministrando entendimiento sobre su funcionalidad.

Por otra parte, les cabe una función singular como fuente de generación de conocimiento científico-tecnológico, que sirva de base para el desarrollo de procesos de innovación. Sin embargo, esto no tiene sustentabilidad en un escenario de trabajo aislado, sino que debe darse en articulación con el sistema productivo, abriendo espacios de aprendizaje compartido que generan sinergias. Desde este punto de vista, el desafío es constituir redes que permitan una articulación 
del sistema productivo local con las instituciones vinculadas a los procesos de innovación.

\section{El desarrollo de redes socioproductivas territoriales para el impulso de competitividad basada en la ciencia, tecnología e innovación}

Las redes son una forma de concebir las diversas interacciones que se producen en la sociedad, en la cual los procesos de comunicación son fundamentales, ya que constituyen el medio central a través del cual los actores determinan y expresan sus intereses y, en el caso de la ciencia, tecnología e innovación, constituyen el canal de dispersión de las innovaciones técnicas. En efecto, la creación de innovación y conocimiento son procesos interactivos en los cuales diferentes agentes y organizaciones intercambian información y cooperan para producir nuevo conocimiento (Lundvall, 2000).

Respecto de los factores que inciden en la construcción de redes entre instituciones de investigación y el sector productivo, resalta la dimensión sistémica de la tecnología, en la cual la cooperación técnica es fundamental. No obstante, una visión contrapuesta sostiene que la construcción de redes para generar y transmitir conocimientos se apoyaría más en la idea de construir esa colaboración técnico-científica para apoyar procesos en atención a las necesidades sociales locales y regionales, es decir, hacer llegar el conocimiento a los sectores que requieren de él (Casas, 2001). En esta perspectiva, las redes se construyen mediante intercambios entre actores que tienen intereses comunes.

No obstante lo descrito, un punto de partida relevante en la comprensión de la redes socioproductivas y su importancia en el desarrollo competitivo basado en la ciencia, tecnología e innovación es la comprensión y gestión de la cadena de valor (Porter, 2002), en función de las características del territorio. Al respecto, la producción se organiza en actividades, construyéndose ventaja competitiva en función del descubrimiento de nuevas formas de realizarlas y articularlas. La producción es algo más que una suma de actividades, puesto que depende del eslabonamiento con el entorno para una 
ventaja competitiva superior. Es decir, importa la gestión de enlaces y articulaciones para la construcción de ventajas competitivas.

Ello pone de manifiesto que la cadena de valor ha de gestionarse como un sistema y no como una colección de partes separadas, ya que constituye una corriente mayor de actividades (Alburquerque, 1995) que incluye cruces de cadenas de valor de proveedores clientes y actores diversos, siendo la competitividad el resultado de una adecuada gestión de todo el sistema, que enlaza las distintas cadenas de valor de los actores territoriales, creándose un mayor valor mediante la optimización y coordinación de éstos.

Por otra parte, importa distinguir además cómo los agentes económicos aprenden y transforman conocimiento genérico en específico. Al respecto, el conocimiento como factor productivo es difícilmente transable, dados los componentes codificados y tácitos que están presentes en él. En el plano del conocimiento codificado, el know what (saber qué) y el know why (saber por qué) son susceptibles de adquirirse en el mercado y, como tales, pueden ser transados fácilmente; no obstante, el conocimiento tácito, el know how (saber cómo) y el know who (saber quién), no lo es. En efecto, el know how es muy difícil de ser imitado y por ello los conocimientos tácitos son muy importantes para generar innovaciones, sin embargo, muy difícil de ser transferidos (Dosi; 1990).

El punto central es que los elementos cruciales del conocimiento siguen siendo específicos y tácitos, presentes en personas, empresas y territorios específicos; por lo demás, el desarrollo del conocimiento codificado y la réplica de los resultados experimentales dependen del conocimiento tácito y del reconocimiento del know who en el campo científico y tecnológico (Yoguel, 2000).

Ello hace explícita la idea de que la competitividad y capacidad de innovación no ocurren en un vacío, sino que dentro de un territorio que cuenta con redes de actores diversos, que pueden llegar a conformar en su estado óptimo un sistema de innovación. En efecto, los sistemas regionales de innovación se constituyen en una compleja configuración de redes de actores territoriales que potencian 
la capacidad de aprendizaje colectivo y difunden innovación a través de la adaptación, generación y/o difusión de avances tecnológicos (Montero et al., 1999).

Lo anterior aparece como una precisión respecto del concepto de "Sistema Nacional de Innovación", cuyo centro de acción se observa de manera agregada en una red de instituciones en el sector público y privado, cuyas actividades e interacciones inician, importan, modifican y difunden nuevas tecnologías, y constituyen una forma de articular empresas, instituciones y actividades que giran en torno a los procesos de innovación y cuyo objetivo es mejorar el concierto entre dichos elementos, aumentando de esta manera su conectividad y fluidez (Malcolm, 1999). Implica la utilización del conocimiento como insumo clave y se basa en la integración del sistema productivo y el sistema científico-tecnológico a través de políticas ad hoc, que promuevan la difusión de innovaciones mediante la creación de espacios de aprendizaje compartido y el fomento del trabajo en red.

Al respecto, es posible identificar tres elementos clave que subyacen en la formación de un medio tecnológico líder (Castells et al., 1994): i) el conocimiento científico y la información como materia prima, ii) la disponibilidad de capital de riesgo sobre la base de la existencia de una capacidad científica y tecnológica aplicable, que constituye el factor primario de un medio innovador, y iii) la disponibilidad de mano de obra científica y tecnológica altamente calificada, generada a partir de fuertes programas de desarrollo en ingeniería, emprendidos por las universidades, que entonces juegan el doble papel de generar conocimiento científico-técnico y, además, suministrar la mano de obra calificada, necesaria para las operaciones empresariales. Sobre estos factores se gestan redes sociales de información, centradas en una cultura de trabajo orientada a la innovación.

Además, se han distinguido elementos como la presencia de "autorregulaciones" y mecanismos de negociación, que impidan que las tensiones competitivas pongan en riesgo la competitividad global en el sistema (Pyke et al., 1992). Es decir, importa el componente colaborativo, en el que participa una amplia variedad de agentes e instituciones que fomentan el sostén y desarrollo del sistema 
competitivo. En otros casos, se ha revelado que la necesidad de alcanzar grandes escalas de operación para competir por volumen pierde importancia frente a la construcción de externalidades positivas complejas (Quintar et al., 1992).

\section{Planteamiento del problema y metodología del estudio de campo}

Como se ha señalado, para generar procesos de innovación que impliquen un mayor desarrollo es clave la articulación de actores que se mueven en el Estado, el mercado, la sociedad civil y las universidades.

En la actualidad se cuenta con una oferta científico-tecnológica universitaria que, en entornos de economías primario-exportadoras, suele carecer de incentivos al desarrollo científico-tecnológico, dado que la gran empresa, en general, demanda tecnología de sus filiales o relaciones tecnológicas en el exterior, en tanto la pequeña y mediana empresa prácticamente no demanda conocimiento científico ni tecnológico. La oferta científica y tecnológica, por su parte, hace especiales esfuerzos para orientarse hacia la base productiva. Sin embargo, se percibe que dicho esfuerzo de investigación y desarrollo, ejecutado por las universidades, ha estado desconectado de los requerimientos de las empresas locales.

Frente a esto, el estudio buscó entregar un diagnóstico de línea base para la gestión regional de la ciencia y tecnología de la Macro Región Centro Sur de Chile (de la VII a la X regiones), partiendo de la premisa de que existe conocimiento y competencias tecnológicas en el sistema regional, pero no hay una iniciativa articulada que conozca, prospecte y construya colectivamente apuestas tecnológicas que vinculen la producción científica con el mundo de las empresas regionales.

En este contexto, la metodología se desarrolla en función de cuatro ejes que determinan las etapas de investigación:

- Identificación de la actual oferta de ciencia y tecnología del Sistema Regional de Ciencia, Tecnología e Innovación. 
- Identificación de las principales cadenas de valor de las economías regionales.

- Diagnóstico estructural de las principales cadenas de valor del territorio.

- Análisis de brechas, sistematización de resultados y generación de propuestas.

\section{El estado actual de la ciencia y tecnología en la Macro Región Centro Sur}

Pese a la ubicación de Chile en 2005 y 2006 en el lugar 27 del ranking de competitividad global elaborado por el Foro Económico Mundial, posicionándose como la economía de mejor desempeño en Latinoamérica, persisten áreas con fuertes debilidades que urge superar. Estas se encuentran en capital humano, inversión en investigación y desarrollo (I+D) y también en su insuficiente diversidad productiva que, según evidencia internacional, es clave para la competitividad y el crecimiento.

La vinculación de las empresas con la actividad científica y tecnológica al servicio de la innovación, a través de consorcios tecnológicos u otros instrumentos similares, es todavía incipiente. La superación de esto se lograría implementando estrategias que permitan una conexión más operativa entre la oferta científica-tecnológica regional con las demandas reales de tecnología e innovación del ámbito productivo público y privado de los sectores económicos clave de las regiones.

De lo anterior se desprende, como requisito esencial, conocer el estado actual de los recursos humanos y físicos dedicados o con potencial de abocarse a actividades de I+D, y evaluar al mismo tiempo el grado de desarrollo de las diversas disciplinas que se asocian a requerimientos de innovación de sectores productivos clave de la región. 


\section{La oferta de ciencia y tecnología en la Macro Región Centro Sur ${ }^{2}$}

Se observan varias áreas con alto grado de desarrollo de la oferta en ciencia y tecnología ( $C \& T)$, que se pueden apreciar por los proyectos de investigación, recursos humanos e infraestructura física disponibles en universidades; existencia de centros de investigación especializados, tanto públicos como privados; montos asociados a proyectos y grados de complejidad de los proyectos, como asimismo la existencia de consorcios, considerados la forma más adecuada de asociar la investigación con la innovación.

Tabla $\mathrm{N}^{\circ}$ 1. Resumen de recursos humanos involucrados en proyectos de investigación en la Macro Región Centro Sur en las áreas definidas

\begin{tabular}{|l|r|r|r|c|}
\hline ÁREAS DISCIPLINARIAS & $\begin{array}{c}\mathrm{N}^{\circ} \\
\text { doctores }\end{array}$ & $\begin{array}{c}\mathrm{N}^{\circ} \\
\text { magíster }\end{array}$ & $\begin{array}{c}\text { No } \\
\text { profesionales }\end{array}$ & Total \\
\hline Forestal & $70(10,31 \%)$ & $35(8,87 \%)$ & $103(17,91 \%)$ & $208(12,61 \%)$ \\
\hline Acuícola y Pesca & $49(7,22 \%)$ & $20(5,06 \%)$ & $46(8,00 \%)$ & $115(6,97 \%)$ \\
\hline $\begin{array}{l}\text { Agroindustria y } \\
\text { C. Silvoagropecuarias }\end{array}$ & $81(11,93 \%)$ & $63(15,95 \%)$ & $70(12,17 \%)$ & $214(12,98 \%)$ \\
\hline $\begin{array}{l}\text { Ciencias de la Tierra y } \\
\text { del Ambiente }\end{array}$ & $23(3,39 \%)$ & $6(1,52 \%)$ & $16(2,78 \%)$ & $45(2,73 \%)$ \\
\hline Biotecnología & $30(4,42 \%)$ & $3(0,76 \%)$ & $20(3,48 \%)$ & $53(3,21 \%)$ \\
\hline Cs. de los Materiales & $67(9,87 \%)$ & $32(8,10 \%)$ & $96(16,70 \%)$ & $195(11,83 \%)$ \\
\hline $\begin{array}{l}\text { Hdes., Cs.Sociales, Ec. } \\
\text { y Administrativas }\end{array}$ & $117(17,23 \%)$ & $158(40,00 \%)$ & $140(24,34 \%)$ & $415(25,17 \%)$ \\
\hline Ciencias Básicas & $242(35,64 \%)$ & $78(19,75 \%)$ & $84(14,61 \%)$ & $404(24,50 \%)$ \\
\hline TOTAL & 679 & 395 & 575 & 1649 \\
\hline
\end{tabular}

Fuente: elaboración propia.

2 Los montos totales presentan una suma inferior a la real por carencia de información respecto de algunos proyectos. No obstante, en niveles relativos, estos datos se presentan como una aproximación de las capacidades científico-tecnológicas del territorio. 
Gráfico $N^{\circ} 1$. Recursos humanos involucrados en proyectos de investigación en la Macro Región Centro Sur, en las áreas definidas

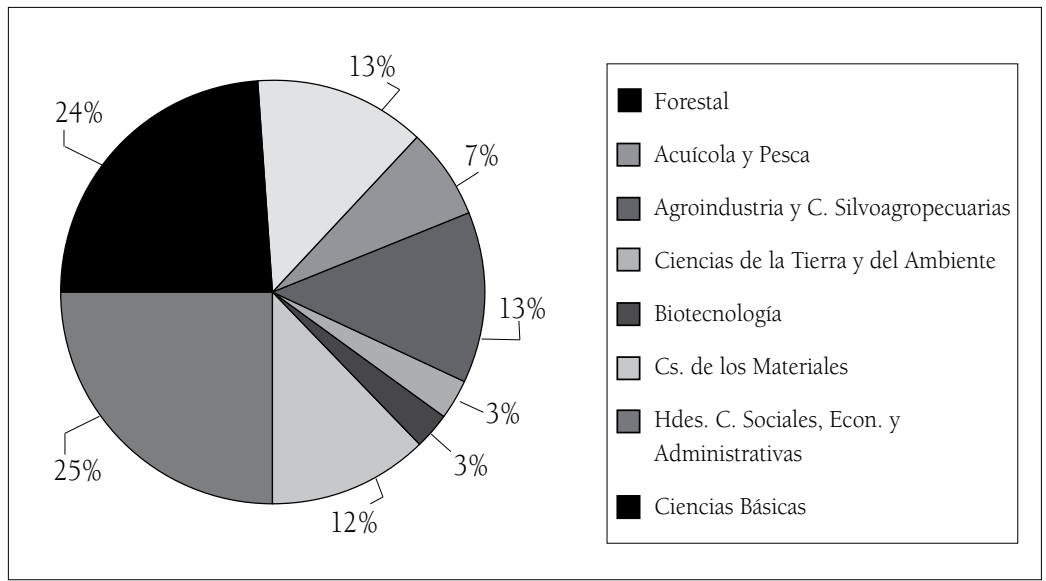

Fuente: elaboración propia.

El área forestal aparece con una importante cantidad de investigadores y doctores después de Ciencia Básica, centros de investigación exclusivos, investigadores en universidades, número de proyectos relevantes. Aunque el número de proyectos en desarrollo es menor que en Agroindustria y Ciencias Silvoagropecuarias, existe mayor diversidad de centros autónomos dedicados a la investigación y mayor compromiso del sector productivo. Otras áreas de buen desarrollo en recursos y montos invertidos en I+D son agroindustria, ciencias silvoagropecuarias y las ciencias de la tierra y el ambiente.

También resulta significativa la participación del área acuícola y pesca, que agrupa un número menos significativo de investigadores que los casos anteriores, pero que, sin embargo, aparece con la segunda cifra en importancia respecto de los montos captados a través de proyectos del total de recursos que fue posible pesquisar en el presente estudio.

Destaca el importante número de investigadores y su proporción ligados al área de las Humanidades, Ciencias Sociales, Económicas y Administrativas. No obstante, ésta se ha desarrollado casi exclusivamente en el ámbito académico, con poca interacción con sectores externos y muestra una ausencia de centros especializados. 
Los investigadores que trabajan en esta área tienen generalmente proyectos poco ligados al entorno y dispersos con un número insuficiente de doctorados, lo cual no permitiría emprender proyectos de mayor relevancia.

El área de Ciencias Básicas, como ocurre en casi todas la universidades nacionales, es la que reúne el número mayor de investigadores y, especialmente, concentra la mayor proporción de doctores, con una gran diversidad de líneas de investigación y de proyectos en ciencias físico-matemáticas, química y biología. El financiamiento a proyectos de ciencia pura y aplicada proviene esencialmente de fondos universitarios internos, de Fondecyt y Fondef 3 .

Por otra parte, existiría un importante equipamiento en áreas que se dedican a I+D, el cual es producto de proyectos concursables ganados, especialmente del tipo Fondef. No obstante, dado la rápida obsolescencia de este tipo de infraestructura, es necesario realizar un estudio adicional de demandas de equipamiento mayor en diversas áreas de investigación de centros y universidades regionales.

Finalmente, cabe destacar que los recursos humanos totales con que cuenta la Macro Región Centro Sur constituye una fortaleza que debería incrementar las actividades de innovación, en la medida en que se incrementen políticas regionales y estrategias que promuevan el acercamiento entre investigadores, empresas y sector público.

Tabla $\mathrm{N}^{0}$ 2. Proyectos de I \& D y principales montos de financiamiento en MM $\$$.

\begin{tabular}{|l|c|c|}
\hline ÁREAS & $\mathrm{N}^{\circ}$ proyectos & Montos MM\$. \\
\hline Forestal & $47(13,62 \%)$ & $3.814(16,40 \%)$ \\
\hline Acuícola y Pesca & $39(11,30 \%)$ & $6.789(29,19 \%)$ \\
\hline Agroindustria y Cs. Silvoagropecuarias & $96(27,83 \%)$ & $6.206(26,68 \%)$ \\
\hline Ciencias de la Tierra y del Ambiente & $26(7,54 \%)$ & $1.512(6,50 \%)$ \\
\hline Biotecnología & $18(5,21 \%)$ & $230(0,98 \%)$ \\
\hline Cs. de los Materiales & $31(8,99 \%)$ & $1.826(7,85 \%)$ \\
\hline Hdes., Cs.Sociales, Ec.y Administrativas & $45(13,04 \%)$ & $1.414(6,08 \%)$ \\
\hline Ciencias Básicas & $43(12,46 \%)$ & $1.468(6,31 \%)$ \\
\hline TOTAL & 345 & 23.259 \\
\hline
\end{tabular}

Fuente: elaboración propia.

3 Fondo Nacional de Desarrollo Científico y Tecnológico y Fondo de Fomento al Desarrollo Científico y Tecnológico. 


\section{Estado actual del sistema económico productivo de la Macro Región Centro Sur}

En este punto se reseñan algunos aspectos clave de la dinámica económica productiva de regiones que componen la Macro Región Centro Sur de Chile, lo que constituye un contexto que describe la línea base económico-productiva del territorio

\section{Respecto del proceso de crecimiento económico en la Macro Región Centro Sur}

En su conjunto, la actividad económica macro regional ha experimentado un crecimiento relativamente débil con relación al resto del país. La excepción corresponde a las regiones de Los Lagos y Los Ríos (recién separadas en dos regiones), que durante los últimos periodos experimentaron juntas un crecimiento notable, por encima de los promedios nacionales. Respecto de sectores de actividad, resalta el caso del sector Pesca, en el cual dichas regiones explican un $54,85 \%$ del PIB generado en escala nacional en el sector, mientras que la Región del Bío-Bío explica un 13,62\%, con lo que el aporte de la macro región al PIB nacional en el rubro supera los dos tercios. Algo similar, aunque de menor magnitud, ocurre en el caso de los sectores electricidad, gas y agua con la Región del Bío-Bío (18,61\%) y la Región del Maule (13,85\%); la industria manufacturera en el caso de la Región del Bío-Bío (17,55\%); y el sector agropecuario silvícola en el caso de las regiones del Maule (13,38\%) y del Bío-Bío (13,29\%). 
Gráfico No 2. Composición sectorial del PIB Macro Región Centro Sur

\begin{tabular}{|l|r|r|r|r|r|r|}
\hline $\begin{array}{l}\text { PIB SECTORIAL MACRO } \\
\text { REGIÓN }\end{array}$ & \multicolumn{1}{|c|}{ VII } & \multicolumn{1}{c|}{ VIII } & \multicolumn{1}{c|}{ IX } & \multicolumn{1}{c|}{ X } & MACRO & \multicolumn{1}{c|}{$\%$} \\
\hline Agropecuario - silvícola & 233.661 & 232.214 & 147.853 & 185.261 & 798.969 & 10,77 \\
\hline Pesca & 2.546 & 73.602 & 415 & 296.415 & 372.978 & 5,03 \\
\hline Minería & 1.224 & 5.738 & 670 & 2.098 & 9.730 & 0,13 \\
\hline Industria manufacturera & 287.330 & 1.085 .081 & 96.273 & 258.126 & 1.726 .810 & 23,27 \\
\hline Electricidad, Gas y Agua & 158.868 & 213.459 & 19.601 & 58.428 & 450.356 & 6,07 \\
\hline Construcción & 101.720 & 291.441 & 115.823 & 169.755 & 678.739 & 9,15 \\
\hline Comercio, restaurantes y hoteles & 115.594 & 284.838 & 96.224 & 135.750 & 632.406 & 8,52 \\
\hline Transporte y comunicaciones & 86.612 & 237.648 & 62.738 & 132.334 & 519.332 & 7,00 \\
\hline $\begin{array}{l}\text { Servicios financieros y } \\
\text { empresariales }\end{array}$ & 86.059 & 252.793 & 71.580 & 97.886 & 508.318 & 6,85 \\
\hline Propiedad de vivienda & 92.027 & 264.686 & 111.716 & 154.209 & 622.638 & 8,39 \\
\hline Servicios personales & 146.545 & 420.127 & 167.477 & 200.866 & 935.015 & 12,60 \\
\hline Administración pública & 44.073 & 113.530 & 56.654 & 67.304 & 281.561 & 3,79 \\
\hline Menos: Imputaciones bancarias & -22.588 & -46.886 & -18.575 & -28.057 & -116.106 & $-1,56$ \\
\hline PIB MACRO REGIÓN & 1.333 .671 & 3.428 .271 & 928.449 & 1.730 .374 & 7.420 .765 & 100,00 \\
\hline \% & 17,97 & 46,20 & 12,51 & 23,32 & 100,00 & \\
\hline
\end{tabular}

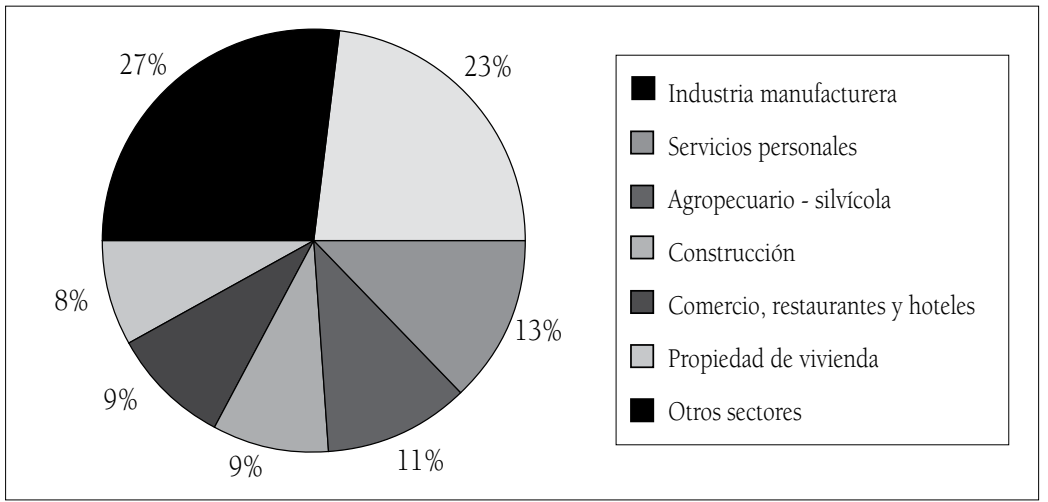

Fuente: elaboración propia basada en información del Banco Central, Chile.

Los sectores descritos son los que en mayor medida aportan al producto macro regional en el periodo. El peso de cada uno de estos rubros se ha mantenido relativamente estable a través del tiempo, habida consideración del sector de la Pesca, localizado en la Región de Los Lagos y Los Ríos, y leves caídas en la participación agropecuaria silvícola, lo que permite prever que, de darse un proceso de reconversión en la estructura productiva macro regional, éste sería un fenómeno paulatino y de largo plazo. 


\section{Respecto del empleo en la Macro Región Centro Sur}

El empleo asociado al proceso de expansión productiva durante la última década en la Macro Región crece a una tasa inferior a lo registrado en el país. Por otro lado, según sectores de actividad, son relevantes en el ámbito nacional los servicios comunales, sociales y personas, que explicaban en 2008 un 27,42\% del empleo nacional; luego, el comercio con un 19,63\%; la industria manufacturera $13,32 \%$, y, finalmente, la agricultura, caza y pesca con $12,00 \%$. En tanto en el plano de la Macro Región destacan los servicios comunales, sociales y personales, con gran importancia en las cuatro regiones bajo estudio, explicando un $25,72 \%$ de la generación de empleo macro regional; luego, la agricultura, caza y pesca, que explica un $23,10 \%$ de él, con gran relevancia en la regiones del Maule (31,30\%), Araucanía (29,22\%) y Los Ríos y Los Lagos (24,49\%). Por otro lado, es relevante consignar que, en función de la importancia que presenta la generación del empleo sectorial en las regiones del territorio centro sur respecto del país, resalta el caso de la agricultura, caza y pesca que se concentra especialmente en la Macro Región.

Gráfico No 3. Composición sectorial del empleo Macro Región Centro Sur

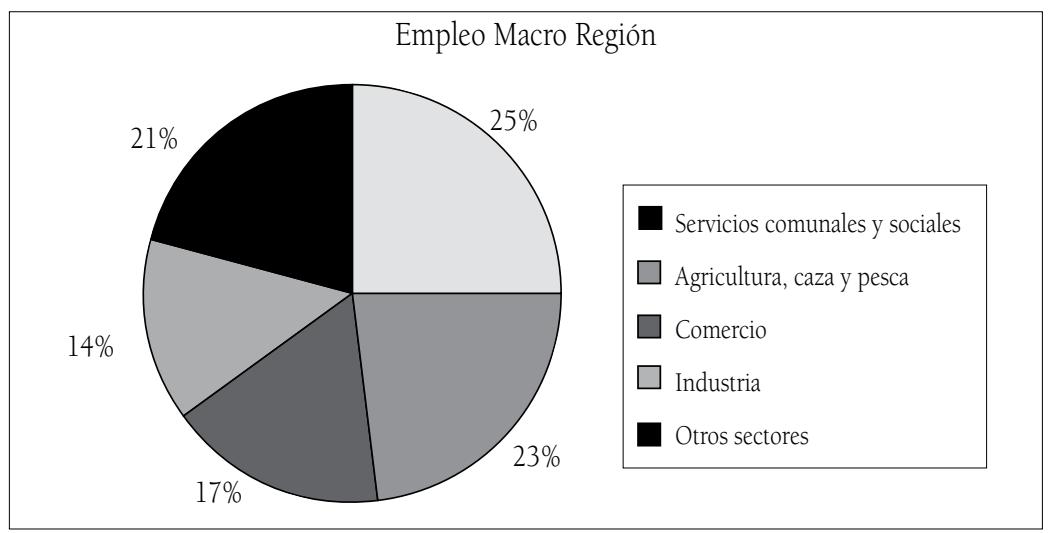




\begin{tabular}{|l|r|r|r|r|r|r|}
\hline EMPLEO SECTORIAL & \multicolumn{1}{|c|}{ VII } & \multicolumn{1}{c|}{ VIII } & \multicolumn{1}{c|}{ IX } & \multicolumn{1}{c|}{ X } & \multicolumn{1}{c|}{ TOTAL } & \multicolumn{1}{c|}{$\%$} \\
\hline Agricultura caza y pesca & 233.661 & 232.214 & 147.853 & 185.261 & 798.969 & 10,77 \\
\hline Minas y canteras & 2.546 & 73.602 & 415 & 296.415 & 372.978 & 5,03 \\
\hline Industria & 1.224 & 5.738 & 670 & 2.098 & 9.730 & 0,13 \\
\hline Electricidad, gas y agua & 158.868 & 213.459 & 19.601 & 58.428 & 450.356 & 6,07 \\
\hline Construcción & 101.720 & 291.441 & 115.823 & 169.755 & 678.739 & 9,15 \\
\hline Comercio & 115.594 & 284.838 & 96.224 & 135.750 & 632.406 & 8,52 \\
\hline Transporte y comunicaciones & 86.612 & 237.648 & 62.738 & 132.334 & 519.332 & 7,00 \\
\hline Servicios financieros & 86.059 & 252.793 & 71.580 & 97.886 & 508.318 & 6,85 \\
\hline Servicios comunales y sociales & 92.027 & 264.686 & 111.716 & 154.209 & 622.638 & 8,39 \\
\hline TOTAL & 146.545 & 420.127 & 167.477 & 200.866 & 935.015 & 12,60 \\
\hline
\end{tabular}

Fuente: elaboración propia basada en información del Banco Central, Chile (id).

Los sectores descritos son los que en mayor medida aportan a la generación de empleo macro regional en la última década. El peso de cada uno de estos rubros se ha mantenido relativamente estable a través del tiempo, habida consideración del incremento sectorial del sector electricidad, gas y agua en las regiones de Los Ríos y Los Lagos, la leve contracción del sector minas y el estancamiento relativo en la generación de empleo en el sector agricultura, caza y pesca.

\section{Respecto de la inserción internacional de la Macro Región Centro Sur}

No obstante el evidente incremento de la actividad exportadora de la Macro Región, se experimenta un rezago en el territorio respecto del dinamismo con el que aumenta la actividad exportadora en el nivel agregado país. Así como la actividad económica macro regional ha experimentado un crecimiento relativamente débil, tal como se ha observado en los puntos precedentes, se consigna además la lentitud del crecimiento exportador de ésta respecto del país.

En términos de sectores, son producciones relevantes la industria de alimentos, que explica un $36,75 \%$ de las exportaciones totales de la Macro Región, con gran importancia en las regiones de Los Ríos y Los Lagos, donde representa un 91,49\% de su producción de exportación, aunque también reflejando porcentajes importantes de exportación de la Región del Maule (20,07\%), Región del Bío-Bío (16,29\%) y Región de La Araucanía (10,59\%). Destaca a su vez la fabricación de celulosa, papel y cartón, que explica un 27,80\% de las producción de exportación, siendo especialmente relevante en 
la Región de La Araucanía, con un 71,46\% de su producción de exportación, en la Región del Bío-Bío donde representa un 37,76\% de las exportaciones y en la Región del Maule, donde abarca un 28,56\% de las exportaciones regionales.

Gráfico No 4. Composición de exportaciones Macro Región Centro Sur

\begin{tabular}{|c|c|c|c|c|c|c|}
\hline EXPORTACIONES US\$FOB 2007 & VII & VIII & IX & $\mathrm{X}$ & MACRO & $\%$ \\
\hline Agricultura & 27.781 & 10.589 & 16.866 & 26.371 & 81.607 & 0,83 \\
\hline Frutas & 340.934 & 48.540 & 15.902 & 14.303 & 419.679 & 4,27 \\
\hline Ganadería & 474 & 1.324 & 129 & 1.714 & 3.641 & 0,04 \\
\hline Madera en pie & 418 & 3.614 & l & 4.852 & 8.885 & 0,09 \\
\hline Extracción de madera & 123 & 4.853 & 84 & 876 & 5.936 & 0,06 \\
\hline Pesca extractiva & 0 & 12.358 & 85 & 1.926 & 14.369 & 0,15 \\
\hline Petróleo y gas natural & 0 & 0 & 0 & 0 & 0 & 0,00 \\
\hline Explotación minas carbón & 0 & 0 & 0 & 0 & 0 & 0,00 \\
\hline Cobre y hierro & 2.343 & 0 & 0 & 0 & 2.343 & 0,02 \\
\hline Resto de minería metálica & 0 & 0 & 0 & 0 & 0 & 0,00 \\
\hline Minería no metálica & 21 & 251 & 11 & 73 & 356 & 0,00 \\
\hline Minería N.C.E.P. & 0 & 502 & 0 & 0 & 502 & 0,01 \\
\hline Industria Alimentos & 273.001 & 880.963 & 45.442 & 2.416 .411 & 3.615 .817 & 36,75 \\
\hline Alimentos forrajeros & 0 & 1.857 & 107 & 10.864 & 12.828 & 0,13 \\
\hline Bebidas, líquidos y alcoholes & 274.747 & 10.583 & 3.325 & 20.490 & 309.145 & 3,14 \\
\hline Tabaco & 0 & 0 & 0 & 0 & 0 & 0,00 \\
\hline Textiles & 129 & 45.553 & 255 & 2.370 & 48.307 & 0,49 \\
\hline Confección prendas de vestir & 2 & 96 & 1 & 10 & 109 & 0,00 \\
\hline Confección prendas de vestir & 11.503 & 21.315 & 932 & 1.987 & 35.737 & 0,36 \\
\hline Calzados & 0 & 155 & 3 & 0 & 158 & 0,00 \\
\hline Forestales & 25.151 & 1.713 .753 & 31.183 & 77.923 & 1.848 .010 & 18,78 \\
\hline Muebles & 2.386 & 1.754 & 6.885 & 78 & 11.103 & 0,11 \\
\hline Fabricación celulosa, papel y cartón & 388.426 & 2.040 .231 & 306.716 & 11 & 2.735 .384 & 27,80 \\
\hline Editoriales e imprentas & 2 & 82 & 0 & 0 & 84 & 0,00 \\
\hline Productos químicos básicos & 6.121 & 66.933 & 35 & 1.050 & 74.139 & 0,75 \\
\hline Productos químicos preparados & 1.502 & 6.991 & 45 & 47.622 & 56.160 & 0,57 \\
\hline Refinación petróleo y productos derivados & 0 & 437.635 & 2 & 340 & 437.977 & 4,45 \\
\hline Cauchos & 0 & 21 & 0 & 0 & 21 & 0,00 \\
\hline Productos de plástico & 286 & 240 & 204 & 610 & 1.340 & 0,01 \\
\hline Productos barro, loza y porcelana & 0 & 4.683 & 0 & 0 & 4.683 & 0,05 \\
\hline Vidrio y manufactura de vidrio & 5 & 10.715 & 1 & 55 & 10.776 & 0,11 \\
\hline Productos mineros no metálicos & 11 & 13 & 0 & 1 & 25 & 0,00 \\
\hline Industria básica fierro y acero & 515 & 13.614 & 202 & 1 & 14.332 & 0,15 \\
\hline Industria básica metales no ferrosos & 2.582 & 1.041 & 428 & 1.919 & 5.970 & 0,06 \\
\hline Productos metálicos & 175 & 30.528 & 54 & 285 & 31.042 & 0,32 \\
\hline Maquinarias y equipos & 1.025 & 2.327 & 228 & 2.016 & 5.596 & 0,06 \\
\hline Material eléctrico & 12 & 182 & 9 & 724 & 927 & 0,01 \\
\hline Material de transporte & 52 & 4.026 & 9 & 4.176 & 8.263 & 0,08 \\
\hline Instrumental médico, óptica, relojería & 6 & 61 & 1 & 33 & 101 & 0,00 \\
\hline Joyería y artículos conexos & 0 & 84 & 0 & 2 & 86 & 0,00 \\
\hline Instrumentos musicales & 0 & 0 & 0 & 0 & 0 & 0,00 \\
\hline Artículos deportivos y atletismo & 0 & 1 & 3 & 0 & 4 & 0,00 \\
\hline Otros productos no especificados & 0 & 0 & 0 & 4 & 4 & 0,00 \\
\hline Productos exhibición cinematográfica & 0 & 0 & 0 & 0 & 0 & 0,00 \\
\hline
\end{tabular}




\begin{tabular}{|l|r|r|r|r|r|r|}
\hline Servicios esparcimiento y diversión & 0 & 0 & 17 & 0 & 17 & 0,00 \\
\hline Otros & 238 & 30.005 & 54 & 2.196 & 32.493 & 0,33 \\
\hline TOTAL & 1.359 .971 & 5.407 .473 & 429.219 & 2.641 .293 & 9.837 .956 & 100,00 \\
\hline$\%$ & 13,82 & 54,97 & 4,36 & 26,85 & 100,00 & \\
\hline
\end{tabular}

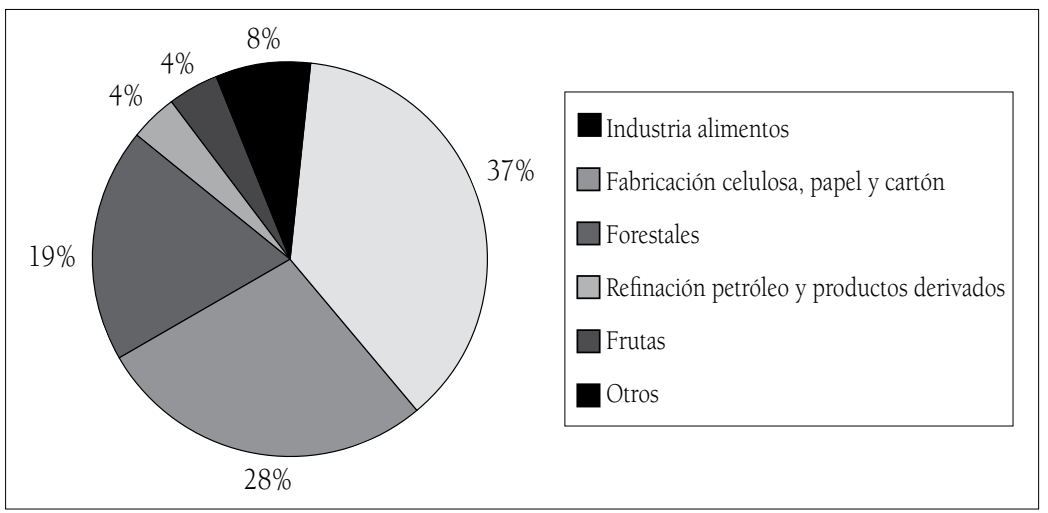

Fuente: elaboración propia basada en información del Banco Central, Chile (Id).

En general, resalta la impresión de una canasta exportadora dominada por la producción primaria o bien por producciones ligadas a la explotación de recursos naturales, con bajos grados de procesamiento, basadas en economías de escala. En efecto, siendo ésta la especialización exportadora que se aprecia en la Macro Región, constituyen a su vez sectores que suelen experimentar una expansión relativa superior en ella, profundizando una especialización exportadora hacia la producción de commodities.

\section{Síntesis del estado del sistema productivo de la Macro Región Centro Sur y sus posibilidades frente al sistema de ciencia y tecnología territorial}

El análisis precedente describe un avance gradual hacia la industrialización y la agregación de valor. En efecto, se evidencia un crecimiento en los envíos sectoriales de bienes industrializados tradicionales y un leve retroceso relativo en la producción de bienes primarios. Lo anterior, por cierto, evidencia el desarrollo productivo hacia la agregación de valor, no obstante la prácticamente inexistente producción de bienes difusores de progreso técnico y el lento crecimiento en la producción de bienes duraderos. 
En el fondo, no obstante el avance industrializador, la prácticamente inexistente producción de bienes difusores de progreso técnico y el estancamiento de la producción de bienes duraderos puede ser una señal de una relativa falta de necesidad de desarrollo científico-tecnológico por parte del sector productivo, que no demandaría de avances técnicos, fundamentalmente por la escasa complejidad de sus procesos productivos.

Por cierto, lo planteado constituye un elemento estratégico cardinal para tomar en cuenta en los análisis del sistema de ciencia, tecnología e innovación de la Macro Región, evidenciado que, no obstante los progresos mostrados en el desarrollo productivo e industrial, los desafíos de avanzar hacia fases más avanzadas de generación de valor persisten, transformándose en un requisito para incentivar la demanda por ciencia y tecnología aplicada al territorio y generada desde el mismo.

\section{Cadenas de valor territorial y sistema de ciencia y tecnología de la Macro Región Centro Sur}

Las conclusiones de este punto obedecen al análisis de la sistematización de información secundaria y de un conjunto de entrevistas realizadas en diversas empresas relacionadas con tres cadenas productivas en las cuales se concentra significativamente la economía Macro Regional: el sector forestal maderero, el sector pesquero industrial y el sector agropecuario. En estos casos, se analizaron las estructuras productivas y las fuentes de su complejidad, y se proyectaron las posibilidades de desarrollo del sistema de ciencia, tecnología e innovación del área.

\section{Visión desde las cadenas de valor respecto del desarrollo del sistema de ciencia, tecnología e innovación territorial}

Este punto constituye la síntesis de las visiones de un conjunto de actores relacionados con las cadenas de valor más relevantes del territorio, respecto del estado del sistema de ciencia, tecnología e innovación territorial. 


\section{- Respecto del sistema de ciencia y tecnología territorial.}

Se aprecia una visión crítica del actual sistema, en la cual se recogen una serie de limitaciones en la aplicación productiva de avances desarrollados, cuya superación tiene como requisito esencial el desarrollo de articulaciones de investigadores y empresarios con perspectivas de mediano y largo plazo.

Abordar estos cambios implica enfrentar la problemática de la estructura de incentivos con que se mueve la lógica de actuación de cada uno de los agentes del mercado de ciencia y tecnología territorial, partiendo con una enseñanza focalizada en los emprendedores y el emprendimiento, e incorporando competencias funcionales para su fomento. Ello incluye la necesidad de dirigir la enseñanza hacia la transmisión de conocimiento aplicado a los requerimientos productivos del territorio, lo que demanda reformar programas de pre y posgrado en los que se incorporen cambios en los currículos y en las metodologías de enseñanza, de manera de lograr un mayor acercamiento entre el ámbito académico y la esfera de la sociedad y sus instituciones. Por cierto, exige además estimular el cultivo de otras áreas disciplinarias en sentido complejo, más allá del ámbito de trabajo de Ciencias Básicas.

En el plano de las instituciones, se demanda descentralización efectiva, de modo que las acciones tengan grado creciente de pertinencia territorial. En efecto, la centralización genera problemas de implementación de las políticas de fomento a la ciencia y tecnología, constituyéndose muy pocos nexos reales con el sector productivo y las líneas de estímulo, ayuda y colaboración, que aparecen complejas e inaplicables a cada realidad territorial. En efecto, los programas existentes no llegan a las empresas que debieran, sino más bien a instituciones intermedias especializadas en la administración de estos fondos. Por ello, se estima como muy relevante la existencia de fondos para I+D regional como una vía para descentralizar recursos con orientación territorial y acompañamiento institucional específico para empresas durante la ejecución de proyectos de I+D.

Y en el plano de los actores privados, es requisito conectarse con quienes desarrollan el conocimiento científico con miras a generar 
productos y procesos con valor agregado. Esto implica incentivar el flujo de recursos para invertir en ciencia y tecnología desde el sector privado, en el cual los incentivos tributarios pueden ser una herramienta clave para fomentar la inversión en I+D en el sector productivo y en una perspectiva de largo aliento.

En el fondo, la visión que impera es que se requiere abordar la problemática del fomento a la ciencia, tecnología e innovación desde una perspectiva compleja, en la cual las actividades de una y otra naturaleza (investigación y desarrollo) se visualicen desde una óptica integrada y se constituyan espacios de aprendizaje mutuo, articulados socialmente. Al respecto, si bien es cierto las políticas públicas de I+D se han orientado paulatinamente a la investigación de desarrollo y a la vinculación universidad-empresa, aún se perciben contradicciones entre las políticas, los organismos encargados de implementarlas y sus marcos de incentivo vinculables ${ }^{4}$. En definitiva, se necesita una transformación mayor que asuma la complejidad para disponer realmente de un "sistema territorial de innovación".

\section{- Respecto de las relaciones entre investigadores y empresas.}

Al describir la relación entre centros de investigación y empresas, los actores observan que ambos existen en mundos separados: la universidad se aprecia encapsulada, concentrándose en el ámbito académico, dejando en segundo plano el área de investigación aplicada; por otro, el sector productivo se ve poco orientado a un desarrollo basado en la innovación y lo que se hace principalmente en las grandes empresas es fruto de trabajos individuales que, a lo sumo, cuentan con el apoyo de consultoras, en su mayoría extranjeras.

4 Se plantea, por ejemplo, que, por una parte, la política nacional de I+D+i (investigación, desarrollo e innovación) propende a lograr una fuerte vinculación entre la comunidad científica, lo público y lo privado que se refleje en proyectos para productos y procesos utilizables, patentes y spin-off. Sin embargo, en contrapartida, las universidades, quizá como únicas instituciones en el país que concentran el capital científico, son evaluadas para recibir el 5\% del Aporte Fiscal Directo casi exclusivamente por su contribución en investigación básica (publicaciones y FONDECYT), dejando de lado los otros elementos ligados a la vinculación con el medio y a la asociación público-privada. 
El sector productivo señala que la universidad suele dar respuestas demasiado complejas a problemas simples, producto de teorización excesiva y de escasez de experiencia práctica, lo que termina resultando en una pérdida de productividad, tiempo y recursos. También, en términos de innovación, la empresa tiende a concentrarse en los resultados de corto plazo, mientras la universidad no suele percibir la premura por la aplicabilidad a la producción de los conocimientos desarrollados. Para resolver esta divergencia entre la universidad y el sector productivo es necesario un mayor contacto del mundo académico con los agentes económicos.

Debido a lo anterior, la empresa prefiere contratar servicios especializados extranjeros, en lugar de recurrir a fuentes nacionales como institutos tecnológicos y universidades. Pero además existen dos razones intrínsecas a la naturaleza empresarial que dificultarían la creación de lazos cooperativos:

- El sistema de ciencia y tecnología en general tiene un enfoque centrado en un sistema concursable y selecciona estudios que arrojan resultados de corto plazo; por tanto, no se consolidan líneas de investigación de largo plazo en instituciones investigadoras. En contrapartida, los mismos estudios, de importancia similar en el extranjero, duplicarían los plazos nacionales, abordando la complejidad que conllevan los tiempos de investigación.

- Por parte del sistema productivo prevalece una mentalidad más bien reactiva que proactiva, priorizando la actualización técnica, con carencia en el abordaje de los riesgos inherentes al avance técnico con miras al liderazgo de mercado, lo que demanda un cambio en la implementación de las políticas de fomento a la I+D con un capítulo especialmente dedicado al desarrollo del capital de riesgo.

A pesar de que la tecnología importada por la empresa nacional demanda recursos monetarios cuantiosos en comparación con lo que cuesta una investigación realizada en centros de investigación locales, las firmas no toman otras alternativas e igualmente lo hacen por las razones recientemente expuestas. 
Se observa que los mayores nexos logrados entre empresa y universidades, en la mayoría de los casos, no van más allá de la realización de prácticas laborales y la incorporación de profesionales provenientes del territorio donde se ubican las plantas productivas, los que actualmente constituyen en forma mayoritaria el recurso humano con que cuentan las organizaciones estudiadas. Se reconoce no obstante que el sector productivo identifica una oferta de profesionales de buen nivel y bien preparada.

- Respecto de posibilidades de futuro del sistema de ciencia y tecnología territorial.

En este plano se identifica la necesidad de un trabajo conjunto y coordinado, como condición de inicio para lograr un estado de desarrollo que considere la ciencia y tecnología en función de la agregación de valor en la producción. En general, se reconoce el potencial y las competencias que poseen las universidades en términos de su capacidad de investigación, sin embargo, éstas se desenvuelven en un marco de limitaciones en la articulación de un mercado científico-tecnológico eficiente.

Por otro lado, se revela una percepción por parte del sistema productivo de que el sistema de ciencia y tecnología no estaría preparado para hacer frente a sus demandas, lo que se agravaría por la relativa falta de disposición del primero para destinar recursos financieros para fines de investigación.

Asimismo, se constatan carencias en la formación de los recursos humanos. Por un lado, frente al hecho de que las empresas en general se encuentran ligadas a procesos rutinarios; por otro, se observan carencias formativas en habilidades orientadas a liderar procesos de innovación de profesionales egresados de universidades, prevaleciendo sus dotes en la ejecución de tareas. En este sentido, se aprecia que las universidades están desaprovechando una diversidad de áreas que, vistas complementariamente entre sí, tienen un amplio potencial de aplicación sobre los sectores productivos, especialmente en lo referido al aporte de las ciencias sociales y su integración a la investigación aplicada en la empresa, así como de sus requerimientos organizacionales y de relaciones con el entorno. 
En ese sentido, se visualiza a la universidad como entidad de incentivo capaz de generar demanda, haciendo uso de sus competencias. Porque la necesidad de innovación está latente en la industria, sin embargo, ésta, no sabe dónde buscarla y es ahí entonces que las relaciones y desarrollo de redes orientadas a satisfacer esa necesidad comunicacional juegan un papel clave.

En definitiva, es preciso identificar las barreras que coartan la conectividad entre los actores. Como no existen puntos de encuentro entre ellos y existe desconocimiento por parte del sector productivo de capacidades y oferta de servicios de investigación de universidades y centros de investigación, es preciso un esfuerzo de estas instituciones por realizar actividades comunicacionales para crear la necesidad de realizar investigaciones conjuntas.

Sin embargo, la posibilidad descrita se complica todavía por las brechas de velocidad de acción que los actores productivos tienden a identificar frente a los procesos de innovación. Por una parte, las instituciones de investigación reaccionan lentamente a los requerimientos y, por otra, las empresas prefieren hacer rápidas inversiones en tecnologías extranjeras, siempre que estén en condiciones financieras para hacerlo. Para hacer frente a esta problemática es necesario construir una visión compartida de largo plazo, recogiendo la importancia de generar externalidades que se derramen al resto del aparato productivo territorial y generen beneficios sistémicos que incrementen la competitividad. Todo lo cual requiere trabajo cooperativo intersectorial y multidisciplinario, junto a gestión de redes socioproductivas.

\section{A modo de conclusiones}

En general, puede aseverarse la persistencia de asimetrías de información entre los actores del sistema, en un marco de descoordinación, desconfianzas e incomunicación. Ello a pesar de reconocerse la necesidad de apoyo científico-tecnológico y de contar con condiciones de inicio y agentes capaces de desarrollarla, pero que, en el contexto de falta de conectividad imperante, coarta las posibilidades de aplicaciones efectivas en el sistema productivo. 
En el marco de lo anterior, se manifiesta la paradoja de que las grandes empresas de los sectores productivos más consolidados del territorio, aparentemente más demandantes de avances técnicos, sean las que relativamente han desarrollado menos vínculos con los actores del sistema científico-tecnológico territorial. A la postre, canalizan sus demandas de avances técnicos en la oferta externa, ferias, giras y conferencias; al contrario de empresas más pequeñas que, frente a un marco de limitaciones para una estrategia en idéntica dirección, se mantienen rezagadas o bien emprendan esfuerzos iniciales en la construcción de vínculos, fundamentalmente mediante apoyos provenientes de la institucionalidad pública.

En consecuencia, se espera que poco a poco emerjan iniciativas de articulación para el desarrollo de la ciencia, tecnología e innovación en el territorio; ello sobre la base de proyectos específicos y creación de centros de estudio especializados, teniendo claro que el proceso será de largo plazo, porque necesita construirse apoyado en un complejo tejido de interacciones y cooperación de variados actores, a objeto de generar conocimiento pertinente al territorio y darle sentido de aplicabilidad, sostenibilidad y fuerza de conjunto.

En este caso, el desafío reside en vincular conocimiento propio a una producción de valor en el territorio -muy señalado aun por la industria primaria y la elaboración de materias primas-, sobre la base de una mirada sistémica que vincule empresa privada, institucionalidad pública y centros de estudio e investigación, asegurando así la provisión de ciencia y tecnología con pertinencia y potencial de aplicabilidad territorial. Es en este contexto en el cual se circunscriben avances notables en el territorio, especialmente en biotecnología y trabajo genético desarrollado en torno a la industria de alimentos, acuícola y procesamiento de madera de alta calidad, en el que precisamente se distingue el crecimiento de la industrialización de los sectores claves de la Macro Región. 
- Respecto de la capacidad del sistema productivo para incentivar el desarrollo de ciencia y tecnología territorial: una visión desde la demanda.

La economía chilena ha experimentado importantes transformaciones en las últimas décadas, señaladas por el traspaso de la actividad productiva al sector privado, en el contexto de una institucionalidad pública focalizada en incentivar la actividad económica y regularla. Este elemento transversal del desarrollo económico chileno caracteriza también su desarrollo científico-tecnológico.

En efecto, junto con el desenvolvimiento económico en función de la inversión privada, se evidencia en Chile un marcado proceso de concentración industrial, especialmente en los principales sectores económicos. En estos mismos sectores, la propia concentración y las estrategias de integración vertical, especialmente hacia atrás, en la pretensión de asegurar insumos productivos, llevan a contar con cadenas de valor cortas y concentradas, lo que limita espacios para el desarrollo de eslabonamientos y surgimiento de nuevas actividades. En efecto, se acotan las posibilidades para el desarrollo de innovaciones en función de nuevas actividades eslabonadas con sectores económicos más consolidados y en mejores condiciones de producir acompañamiento de mercado para dichas innovaciones.

A lo anterior se agrega la madurez y bajo grado de complejidad que, en general, caracteriza la canasta productiva chilena, muy señalada por la elaboración de productos con bajo grado de complejidad, generalmente materias primas, que compiten por costo en mercados internacionales. Estos mismos procesos, por su reducida complejidad, presentan una baja capacidad de tensión al sistema científico tecnológico territorial y, a la postre, al operar con tecnologías en general maduras y estandarizadas, desarrollan nexos más eficientes con sus filiales o bien con oferentes tecnológicos externos.

No obstante, a pesar de la prevalencia de esta componente de especialización primaria y manufacturera de baja complejidad de la economía territorial, surgen experiencias de aglomeraciones económicas que incentivan y demandan desarrollo científico y tecnológico en el territorio. Tales son los casos de la industria 
alimenticia, acuicultura y madera de calidad con destino a mercados externos. En torno a la cadena forestal maderera, pesquera y agropecuaria se distinguen focos de procesos de industrialización, entre los cuales es posible e interesante pensar en el desarrollo de vínculos de investigación y aprovechar potenciales espacios de aplicación en torno a su eslabonamiento.

Sin embargo, esto demanda abordar la problemática de la carencia de una estrategia tecnológica "formal" incorporada en las empresas, que en general innovaran de manera incremental, obviamente por el componente de riesgo que significa abordar innovaciones de índole más radical. No obstante, aun el proceso de innovaciones incrementales sería acotado porque éste, que ha de verse impulsado generalmente por las condiciones de la demanda, en el caso de los principales sectores económicos del territorio, se entramparía por una demanda local poco exigente y en la cual el "tiraje" proviene principalmente por estímulos externos, de mercados lejanos a la realidad y potencialidad productiva territorial.

En el mismo plano de la estrategia tecnológica de las empresas se debe abordar la problemática de difusión de innovación; desde la gran empresa hacia la mediana y pequeña y su efectivo acompañamiento de mercado, que incluye una no resuelta. En efecto, tan significativa como la desarticulación empresarial entre la gran, mediana y pequeña empresa, son las brechas que se generan entre el avance técnico generado por la gran empresa inserta en los mercados internacionales y las empresas de menor tamaño que no logran conectarse con ellos.

Al respecto -relaciones proveedor-cliente-, con articulación más estrecha entre gran empresa exportadora y mediana empresa proveedora de servicios de aquella es posible construir condiciones favorables para el desarrollo de un sistema productivo con aplicaciones y difusión de innovación hacia redes de pequeñas empresas.

En el fondo, se trata de una estrategia para el desarrollo del sistema territorial de innovación que parte desde el desenvolvimiento de los sectores productivos clave del territorio, los cuales, junto con desenvolverse productivamente, paulatinamente han de ir 
demandando y exigiendo al sistema de ciencia y tecnología territorial. Todo ello implicaría fomentar la gestación y fortalecimiento de pirámides productivas sobre la base de la gran empresa y su articulación estratégica con medianos y pequeños establecimientos del territorio, cuya finalidad sea el desarrollo de mercados proveedores y servicios conexos orientados al desarrollo de innovaciones, abriendo espacios para la ciencia y tecnología aplicada a la realización en los nuevos eslabonamientos productivos que se gesten.

Constituye ésta una estrategia de doble propósito: abrir oportunidades para el desarrollo productivo de la pequeña y mediana empresa y, a su vez, desarrollar el sistema de ciencia y tecnología aplicada del territorio, con efectivo acompañamiento de mercado. Se trata en el fondo de nivelar oportunidades de acceso a mercados, financiamiento, orientación técnica y desarrollo de proyectos de investigación aplicada, recogiendo las limitaciones de acceso que la pequeña y mediana empresa suelen encontrar para su progreso innovativo sobre la base del avance de la ciencia y tecnología territorial.

\section{- Respecto de la capacidad del sistema de ciencia y tecnología para incentivar el desarrollo del sistema productivo territorial: una visión desde la oferta}

Persisten brechas para que la relación universidad-empresa tenga mayor influencia en los procesos de innovación. En efecto, las empresas no cuentan con respaldo de la capacidad científica territorial. De otra manera, la vinculación en los procesos productivos con relación a lo que requieren las empresas no está siendo considerada.

Por otro lado, como se carece de un mercado científicotecnológico desarrollado en la Macro Región, no se han desplegado lineamientos específicos al territorio para el desarrollo del mercado de innovación, en un contexto donde imperan marcos de incentivos nacionales, en general no discriminantes frente a la diversidad territorial y que, por lo demás, se visualiza desconectado de los requerimientos del sistema productivo en general, al privilegiar el fomento por la productividad estrictamente científica (investigación básica, separada de la aplicada), sin considerar paralelamente la 
necesidad de vincular el desarrollo de la ciencia y tecnología a la investigación aplicada.

A la postre, este contexto actúa como un incentivo que, si bien promueve y canaliza fondos para el progreso de la investigación científica, ésta termina desarrollándose de manera encapsulada y aún con bajo grado de interrelación con los requerimientos productivos del territorio.

En este sentido, pensar estrategias relacionadas a la implementación de observatorios prospectivos de ciencia, tecnología e innovación por parte de las universidades, que tengan como principal finalidad monitorear constantemente los requerimientos por parte del sector productivo y promover la búsqueda de respuestas por parte de los equipos científicos frente a éstos, aparece como un vector relevante ante al mayor encapsulamiento relativo que se visualiza en el trabajo investigativo. A su vez, colabora a minimizar el desconocimiento de las capacidades y ofertas de servicios de universidades e instituciones de investigación por parte del sector productivo. Como objetivo general, implicaría disminuir la brecha y diferenciales de velocidad de acción entre oferentes y demandantes en relación con los procesos de innovación de los actores.

- Respecto de la potencialidad institucional para el desarrollo de sistemas territoriales de innovación.

No obstante las iniciativas incipientes y los recursos asociados a ellas, el desafío se relaciona tanto con fomentar el desarrollo de la ciencia y tecnología como también, fundamentalmente, con desarrollar marcos de incentivos pertinentes para un sistema de ciencia y tecnología funcional al desarrollo productivo regional y así avanzar a una fase centrada en la competitividad territorial.

Esto incluye demandas en descentralización que permitan el diseño (y rediseño) de instrumentos para hacerlos pertinentes a los sectores productivos de cada territorio, dentro de un marco de definición de políticas regionales coherente con las potencialidades territoriales. Implica a su vez asignar un papel central a las universidades regionales, que no sólo habrán de intervenir en la 
generación de recursos humanos capacitados y focalizados para los sectores productivos clave, sino que también, fundamentalmente, en enfocar un esfuerzo especial para orientar la investigación básica hacia campos de aplicación relacionados a los requerimientos del sistema productivo.

Por cierto, el fomento de lo anterior demanda el rediseño de un conjunto de marcos de incentivos para el desarrollo de ciencia y tecnología, puesto que hasta hoy imperan aquellos que privilegian el fomento de la productividad científica mediante proyectos de investigación básica y publicaciones, sin considerar necesariamente el desarrollo de vínculos y el ejercicio de la ciencia y tecnología a la investigación aplicada.

Por otro lado, resulta relevante incentivar la inversión en infraestructura tecnológica público-privada-académica en el territorio. Al respecto, ha surgido una serie de iniciativas que han incrementado el potencial científico y tecnológico del territorio. No obstante, aún se percibe muy desconectadas a las empresas en su gestación, lo que nuevamente demanda identificar marcos de incentivos que promuevan su desenvolvimiento. Se trata en el fondo de que estos organismos se constituyan en espacios para desarrollar conocimiento aplicado y aprendizaje conjunto en el territorio, en una entidad en la cual se conectan requerimientos y ofertas de innovación. A su vez, que en su desenvolvimiento se compartan conocimientos, recogiendo la problemática del know how y know who en la gestión del conocimiento competitivo. En este campo, el aprendizaje será la resultante de la capacidad que empresas y universidades en conjunto, con apoyo de instrumentos públicos específicos, tengan para reconocer las necesidades del sistema productivo territorial y enfocarse hacia la creación de conocimiento explícito aplicado a él.

Participaron en el estudio Sergio Moffat, como jefe del equipo consultor, Justo Lisperguer y Ariel Yévenes, como consultores del equipo base, y Luzgarda del Valle, Pía Krago, Roberto Mendiburo, Susana Riquelme, Miriam Troncoso y Gonzalo Valenzuela, como consultores del estudio exploratorio. 


\section{Referencias bibliográficas}

Alburquerque, F. (1995) Competitividad Internacional, Estrategia Empresarial y Papel de las Regiones. Documento 95/28, Serie Ensayos, Dirección de Políticas y Planificación Regional, Instituto Latinoamericano y del Caribe de Planificación Económica y Social (ILPES), abril de 1995.

Casas, R. (2001) El Enfoque de Redes y Flujos de Conocimientos. El Análisis de las Relaciones entre Ciencia, Tecnología y Sociedad. México: Instituto de Investigaciones Sociales, UNAM.

Castells, M. y Hall, P. (1994) Tecnópolis del Mundo. La Formación de los Complejos Industriales en el Siglo XXI. Madrid, España: Alianza Editorial.

CEPAL (1992) El Comercio de Manufacturas en América Latina: Evolución y Estructura 1962-1989. Santiago de Chile: Estudios e Informes de la CEPAL, $\mathrm{N}^{\circ} 88$.

Dosi, G.; Pavitt, K. y Soete L. (1990) The Economics of Technical Change and International Trade. Londres: Harvester Wheatsheaf.

Esser, K.; Hillebrand, W.; Messner, D. y Meyer-Stamer, J. (1994) Competitividad Sistémica. Competitividad Internacional de las Empresas y Políticas Requeridas. Estudios e Informes 11/1994. Instituto Alemán de Desarrollo.

Galdames, R.; Gatica, F; Menéndez, J. y Yévenes, A. (2002) Crisis en la Pesca Industrial. La Necesidad de desenclavar un Sector. En Falabella, G. y Galdames, R. (eds.) Repensar el Desarrollo Chileno. País, Territorio, Cadenas Productivas. Concepción: Ediciones Universidad del Bío-Bío, julio 2002, pp. 370-429.

Gatica, F. (2002) Territorio, Gobierno Local y Circuitos Económicos: el Caso de Coelemu. En Falabella, G. y Galdames, R. (eds.) Repensar el Desarrollo Chileno. País, Territorio, Cadenas Productivas. Concepción: Ediciones Universidad del Bío-Bío, julio 2002, pp. 285-358.

Gay, A. (2002) La Ciencia, la Técnica y la Tecnología. Buenos Aires-Argentina: Tecno Red Educativa, INET, Serie Educación Tecnológica, № 1, 2002, pp. 77-91.

Lundvall, B.A. (2000) The Learning Economy: Some Implications for the Knowledge Base of Health and Education Systems. Knowledge Management in the Learning Society. Education and Skills, OECD, Paris, pp. 125-141.

Malcolm, D. (1999) Sistema Nacional de Innovación: una Aproximación. Revista de la Escuela de Economía y Negocios, Argentina. 
Menéndez, J. (2002) El Casillero Vacío del Fomento Productivo Chileno. En Falabella, G. y Galdames, R. (eds.) Repensar el Desarrollo Chileno. País, Territorio, Cadenas Productivas. Concepción: Ediciones Universidad del Bío-Bío, julio 2002, pp. 95-180.

Millán, F. (1994) Competitividad Internacional de Regiones. Documento 94/22, Serie Ensayos. Dirección de Políticas y Planificación Regionales. Instituto Latinoamericano y del Caribe de Planificación Económica y Social, ILPES.

Montero, C. y Morris, P. (1999) Territorio, Competitividad Sistémica y Desarrollo Endógeno. Metodología para el Estudio de los Sistemas Regionales de Innovación. En Instituciones y Actores del Desarrollo Territorial en el Marco de la Globalización. Concepción: Ediciones Universidad del Bío-Bío e Instituto Latinoamericano y del Caribe de Planificación Económica y Social, pp. 321-374.

Muñoz, O. (2001) Estrategias de Desarrollo en Economias Emergentes. Lecciones de la Experiencia Latinoamericana. Santiago de Chile: Facultad de Ciencias Físicas y Matemáticas, Departamento de Ingeniería Industrial, Universidad de Chile, Facultad Latinoamericana de Ciencias Sociales (FLACSO).

Pérez, C. (2001) Cambio Tecnológico y Oportunidades de Desarrollo como Blanco Móvil. Revista de la Cepal, 75, pp. 115-136.

Polanyi, M. (1962) Personal Knowledge. Towards a Post-Critical philosophy. Nueva York: Harper Torchbooks.

Porter, M. (1979) How Competitive Forces Shape Strategy. Harvard Business Review, 57(2), pp. 137-145.

Porter, M. (1987) Ventaja Competitiva: Creación y Sostenimiento de un Desempeño Superior. $1^{\text {era }}$ edición española. México: CECSA.

Porter, M. (1991) La Ventaja Competitiva de las Naciones. Buenos Aires, Argentina: Javier Vergara Editor.

Porter, M. (1996) What is Strategy? Harvard Business Review, 74(6), pp. 61-78.

Porter, M. (1999) Ser Competitivos: Nuevas Aportaciones y Conclusiones. Bilbao, España: Editorial Deusto.

Porter, M. (2002) Estrategia Competitiva: Técnicas para el Análisis de los Sectores Industriales y de la Competencia. Edición revisada, México: Grupo Patria Cultural S.A. de C.V.

Pyke, F; Becattini. G. y Sengenberger, W. (1992) Los Distritos Industriales y Las Pequeñas Empresas. Distritos Industriales y Cooperación Interempresarial 
en Italia. Madrid, España: Centro de Publicaciones, Ministerio del Trabajo y Seguridad Social.

Quintar, A. y Gatto F. (1992) Distritos Industriales Italianos. Experiencias y Aportes para el Desarrollo de Políticas Industriales Locales. Buenos Aires, Argentina: Documento de Trabajo PRIDE n 29, publicación CEPAL, LC/BUE./R.173.

Ramos, J. (1998) Una Estrategia de Desarrollo a partir de los Complejos Productivos en torno a los Recursos Naturales. Revista de la Cepal, 66.

Rojas, C. (2001) Globalización y Capacidades Colectivas de Aprendizaje Tecnológico. Requerimientos Institucionales a partir del Caso de la Región del Bío-Bío. En Muñoz, O. (ed.) Más allá del Bosque: Transformar el Modelo Exportador. Santiago de Chile: Facultad Latinoamericana de Ciencias Sociales (FLACSO), pp. 145-183.

Yoguel, G. (2000) Creación de Competencias en Ambientes Locales y Redes Productivas. Revista de la Cepal, 71, pp. 105-119.

Recibido : 20 de abril de 2009

Aceptado: 1 de junio de 2009 